\title{
G-Protein-Coupled Receptor Kinases in Hypertension
}

\author{
Daniela Carnevale $\cdot$ Giuseppe Lembo
}

Published online: 13 April 2013

(c) Springer International Publishing Switzerland 2013

G-protein-coupled receptors (GPCRs) are one of the most important classes of proteins in living organisms. The pioneer studies of Brian Kobilka (from Stanford University) and Robert Lefkowitz (from Duke University) led to breakthroughs that have opened the way to a detailed dissection of the mechanisms of GPCR function and made them recently earn the 2012 Nobel Prize in Chemistry.

Notably, the class of proteins and their physiological importance makes this prize extremely well deserved and this has been one of the fastest discovery-to-prize transitions in recent decades. GPCRs provide a superb example of a molecular machine whose subtle workings we have only started to understand. There is a variety of ways in which GPCRs are regulated, activated and inactivated and G-protein binding is just one of them. A recently highlighted class of proteins called arrestins, for instance, can deactivate GPCRs when they are overstimulated and internalize them for degradation [1]. Thus, what is emerging is that molecules that are functionally linked to these proteins and are intertwined to regulate and transduce their functions, can come into manifold types.

D. Carnevale · G. Lembo

Department of Angiocardioneurology, IRCCS Neuromed,

Pozzilli, Isernia, Italy

D. Carnevale · G. Lembo ( $\square)$

Department of Molecular Medicine, "Sapienza" University

of Rome, c/o IRCCS Neuromed, 86077 Pozzilli (IS), Italy

e-mail: giuseppe.lembo@uniroma1.it; lembo@neuromed.it
The review article by Santulli et al. [2], published in this issue, explores this intriguing subject. The authors extensively review the role of one pivotal signalling pathway linked to GPCR and strictly linked to cardiovascular diseases: G-protein-coupled receptor kinases (GRKs).

GRKs are found in nearly all mammalian cells but possess broad and diverse effects ranging from GPCR signal termination to transcriptional regulation [3]. In particular, GRKs terminate the G-protein signalling by phosphorylating the intracellular domains of the receptor and increasing the affinity for arrestin proteins. This regulation allows the receptors to be uncoupled from GPCRs for internalization.

What sounds most interesting is that, among the various human pathophysiologies correlated with hyper-/hypoactivity of GRKs, cardiovascular disease comes to light regarding the intriguing perspective of modulating the expression and/or activity of specific GRKs and their interacting partners as a novel therapeutic strategy.

The GRK family is composed of different members among which GRK2 seems the most important, being the homozygous GRK2-deficient mice embryonic lethal [4]. As extensively reviewed by Santulli et al. [2], the pathophysiological relevance of this isoform in hypertension is revealed by the multitude of studies conducted to discriminate its roles in the different components that may be involved in its onset and end organ damage, namely heart, vasculature, kidney and adrenal gland. Remarkably, the pathophysiological link between hypertension and GRK2 cannot neglect the mounting evidence suggesting a role for this molecule in metabolism regulation, particularly in insulin resistance, a non-trivial aspect often associated with hypertension. Furthermore, the work conducted by the authors has enabled insight into the molecular mechanisms demonstrating the importance of GRK2 in the pathology of insulin resistance. 
Finally, we conclude this perspective on another interesting issue raised by Santulli et al. that points to the possibility that peripheral blood lymphocytes may mirror changes in GRK2 expression/function in other organs, in several pathophysiological conditions. Further studies should explore the possibility of GRK2 as a signalling pathway at the crossroads between cardiovascular diseases and immunity.

Acknowledgments No sources of funding were used to assist in the preparation of this article. The authors have no conflicts of interest that are directly relevant to the content of this article.

Conflict of interest None.

\section{References}

1. Pierce KL, Lefkowitz RJ. Classical and new roles of beta-arrestins in the regulation of G-protein-coupled receptors. Nat Rev Neurosci. 2001;2(10):727-33.

2. Santulli G, Trimarco B, Iaccarino G. G-Protein-Coupled receptor kinase 2 and hypertension: molecular insights and pathophysiological mechanisms. High Blood Press Cardiovasc Prev 2013;2(1). doi:10.1007/s40292-013-0001-8.

3. Kamal FA, Travers JG, Blaxall BC. G Protein-coupled receptor kinases in cardiovascular disease: why 'where' matters. Trends Cardiovasc Med. 2012;22(8):213-9.

4. Jaber M, Koch WJ, Rockman H, et al. Essential role of betaadrenergic receptor kinase 1 in cardiac development and function. Proc Natl Acad Sci USA. 1996;93(23):12974-9. 